\title{
Developmental coupling of cerebral blood flow and fMRI fluctuations in youth
}

Erica B. Baller, M.D., M.S. ${ }^{\mathrm{a}, \mathrm{b}}$, Alessandra M. Valcarcel, Ph.D. ${ }^{\mathrm{c}, t}$, Azeez Adebimpe, Ph.D. ${ }^{\mathrm{a}, \mathrm{b}}$, Aaron Alexander-Bloch, M.D., Ph.D. ${ }^{\text {, }}$ Zaixu Cui, Ph.D. ${ }^{\mathrm{a}, \mathrm{b}}$, Ruben C. Gur, Ph.D. ., ,f, Raquel E. Gur, M.D., Ph.D. ${ }^{\text {bd,f }}$, Bart L. Larsen, Ph.D. ${ }^{\text {a,b }}$, Kristin A. Linn, Ph.D. ${ }^{c}$, Carly M. O’Donnell, B.A. ${ }^{\mathrm{c}}$, Adam R. Pines, B.A. ${ }^{\mathrm{a}, \mathrm{b}}$, Armin Raznahan, Ph.D. ${ }^{\mathrm{e}}$, David. R. Roalf, Ph.D. ${ }^{\mathrm{b}}$, Valerie J. Sydnor, B.A. ${ }^{\mathrm{a}, \mathrm{b}}$, Tinashe M. Tapera, M.S. ${ }^{\mathrm{a}, \mathrm{b}}$, M. Dylan Tisdall, Ph.D. ${ }^{\mathrm{f}}$, Simon Vandekar, Ph.D. ${ }^{\mathrm{g}}$, Cedric H. Xia, M.D., Ph.D. ${ }^{\mathrm{a}, \mathrm{b}}$, John A. Detre, M.D. ${ }^{\mathrm{d}}$, Russell T. Shinohara, Ph.D. ${ }^{\mathrm{c}, \mathrm{h} *}$, Theodore

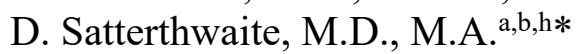

a Penn Lifespan Informatics and Neuroimaging Center (PennLINC)

${ }^{b}$ Department of Psychiatry, University of Pennsylvania

${ }^{c}$ Penn Statistics in Imaging and Visualization Center (PennSIVE), Department of Biostatistics, Epidemiology, and Informatics, University of Pennsylvania

${ }^{\mathrm{d}}$ Department of Neurology, University of Pennsylvania

${ }^{e}$ National Institute of Mental Health, NIH, IRP

${ }^{\mathrm{f}}$ Department of Radiology, University of Pennsylvania

g Department of Biostatistics, Vanderbilt University

${ }^{\mathrm{h}}$ Center for Biomedical Image Computing and Analytics (CBICA)

${ }^{+}$Currently employed by Genentech

*co-senior authors who contributed equally

Conflict of interest: The authors declare no competing financial interests.

Acknowledgements: This work was supported by grants from the National Institute of Mental Health (NIMH; Grant Numbers: R01MH112847 to TDS and RTS; R01MH120482, and R01MH113550 to TDS; R01 MH123550-01 to RTS; R01MH107235 to RCG; 2T32MH019112-29A1 to EBB; T32MH014654 to BLL; F31 MH123063-01A1 to ARP; R01MH120174, R01MH119185, and R56AG066656 to DRR; DGE-1845298 to VJS. The PNC was funded by RC2 grants MH089983 and MH089924 to REG from the NIMH.

Corresponding author:

Theodore D. Satterthwaite, M.D.

Richards Building, 5th Floor, Suite 5A

3700 Hamilton Walk

Philadelphia, PA 19104-6085

Email: sattertt@pennmedicine.upenn.edu 


\section{ABSTRACT}

To support brain development during youth, the brain must balance energy delivery and consumption. Previous studies in adults have demonstrated high coupling between cerebral blood flow and brain function as measured using functional neuroimaging, but how this relationship evolves over adolescence is unknown. To address this gap, we studied a sample of 831 children and adolescents (478 females, ages 8-22) from the Philadelphia Neurodevelopmental Cohort who were scanned at 3T with both arterial spin labeled (ASL) MRI and resting-state functional MRI (fMRI). Local coupling between cerebral blood flow (CBF, from ASL) and the amplitude of low frequency fluctuations (ALFF, from fMRI) was first quantified using locally weighted regressions on the cortical surface. We then used generalized additive models to evaluate how CBF-ALFF coupling was associated with age, sex, and executive function. Enrichment of effects within canonical functional networks was evaluated using spin-based permutation tests. Our analyses revealed tight CBF-ALFF coupling across the brain. Whole-brain CBF-ALFF coupling decreased with age, largely driven by coupling decreases in the inferior frontal cortex, precuneus, visual cortex, and temporoparietal cortex $\left(p_{f d r}<0.05\right)$. Females had stronger coupling in the frontoparietal network than males $\left(p_{f d r}<0.05\right)$. Better executive function was associated with decreased coupling in the somatomotor network $\left(p_{f d r}<0.05\right)$. Overall, we found that CBF-ALFF coupling evolves in development, differs by sex, and is associated with individual differences in executive function. Future studies will investigate relationships between maturational changes in CBF-ALFF coupling and the presence of psychiatric symptoms in youth. 
SIGNIFICANCE: The functions of the human brain are metabolically expensive and reliant on coupling between cerebral blood flow and neural activity. Previous neuroimaging studies in adults demonstrate tight physiology-function coupling, but how this coupling evolves over development is unknown. Here, we examine the relationship between blood flow as measured by arterial spin labeling and the amplitude of low frequency fluctuations from resting-state magnetic resonance imaging across a large sample of youth. We demonstrate regionally specific changes in coupling over age and show that variations in coupling are related to biological sex and executive function. Our results highlight the importance of CBF-ALFF coupling throughout development; we discuss its potential as a future target for the study of neuropsychiatric diseases. 


\section{INTRODUCTION}

The functions of the human brain are metabolically expensive: despite only weighing 1.5 $\mathrm{kg}$ on average, the brain comprises a disproportionate one-fifth of bodily energetic requirements (1-3). To meet such large metabolic demands, the brain receives $20 \%$ of cardiac output $(4,5)$. In healthy subjects, the relationship between brain activity and cerebral blood flow (CBF), or neurovascular coupling, is tightly linked at the local level (6-8). Under normal circumstances, metabolites produced during neuronal activity cause vasodilation in the microvasculature, and thus a localized increase in blood flow, to increase glucose and oxygen delivery to active cells $(6,9)$. By coupling metabolic demand (neural activity) and supply (blood flow), the neurovascular unit maintains appropriate energy balance (9).

To measure neurovascular coupling in vivo, proxies for both localized blood flow and regional brain activity are required. Neurovascular coupling has thus been most frequently characterized by relating two neuroimaging-derived measures: CBF and the amplitude of low frequency fluctuations in resting-state blood oxygen level dependent (BOLD) fMRI (ALFF; 1012). CBF can be measured reliably and without radiation exposure using arterial spin labeled (ASL) MRI (11-13), producing a quantitative measure of blood supply. Although BOLD signal partly reflects changes in $\mathrm{CBF}$, low amplitude fluctuations as measured by ALFF are thought to represent spontaneous brain activity and to underlie intrinsic functional connectivity (14), providing a non-invasive proxy of neuronal function $(15,16)$.

Neurovascular coupling as measured by CBF-ALFF changes in aging, with younger adults displaying higher coupling than healthy older adults $(9,10)$. Sex differences in coupling in adults have also been described (17). Furthermore, decreases in the typically strong coupling between CBF and ALFF have been reported in degenerative and metabolic diseases $(9,18,19)$. 
Notably, these diseases are associated with significant cognitive impairments, further highlighting the importance of the integrity of this coupling relationship.

Despite this growing literature, notable gaps remain. First, prior studies have examined the relationship between $\mathrm{CBF}$ and ALFF primarily at the whole-brain level, yielding a global measure of coupling (20). While informative, such an approach obscures potentially important regional variation in the coupling between CBF and ALFF. Second, previous studies have focused on neurovascular coupling only in healthy and medically ill adults $(9,18,19,21)$. To our knowledge, no prior research has explored how the relationship between blood flow and brain function evolves during childhood, adolescence, and young adulthood. Thus, it is presently unclear how the balance between metabolic demand and supply changes as neurodevelopment unfolds.

Here, we sought to define the local relationship between CBF and ALFF on a regional basis, and to determine how CBF-ALFF coupling evolves over development. To do this, we capitalized on data from the Philadelphia Neurodevelopmental Cohort: a large scale, communitybased study of brain development that included both ASL MRI and fMRI $(22,23)$. We characterized the CBF-ALFF relationship using recently developed tools for inter-modal coupling analysis that allow us to describe the local relationships between these two imaging modalities $(24,25)$. As described below, we extend prior findings demonstrating that blood flow and neural function are strongly related across the brain. Importantly, we also describe how CBF-ALFF coupling evolves in youth, differs by sex, and is related to executive function. 


\section{MATERIALS AND METHODS}

\section{Experimental Design}

\section{Participants}

Participants were drawn from the Philadelphia Neurodevelopmental Cohort (PNC). As previously described, a total of 9,498 participants aged 8-22 years received cognitive assessment and clinical phenotyping, and a subset of 1,601 youths also completed neuroimaging as part of the PNC $(22,26)$. For this report, we excluded participants with missing data, participants currently being treated with psychoactive medication, individuals with medical disorders that could impact brain function, and participants with poor image quality (see below). Eight-hundred thirty-one subjects met criteria and were included in the study (Figure 1). The average age of participants was 15.6 years (standard deviation $(S D)=3.4)$. Forty-two percent $(n=353)$ were male and $58 \%$ were female $(n=478)$. The institutional review boards of both the University of Pennsylvania and the Children's Hospital of Philadelphia approved all study procedures.

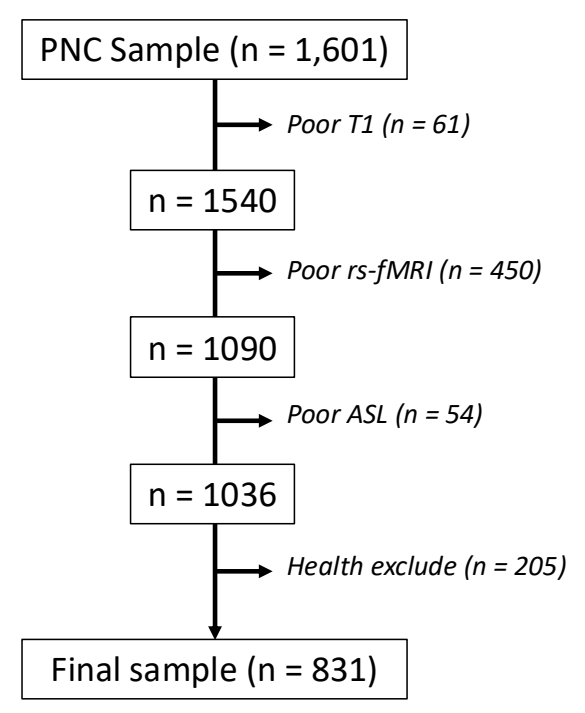

Figure 1. Sample construction. 1,601 participants had neuroimaging scans acquired as part of the PNC. A total of 831 participants were included in the study after excluding participants who failed rigorous quality assessment for poor T1 quality $(\mathrm{n}=61)$, resting state fMRI quality $(\mathrm{n}=$ $450)$, ASL quality $(n=54)$, and medical and psychiatric comorbidities $(n=205)$. 


\section{Cognitive assessment}

Cognition was assessed using the University of Pennsylvania Computerized Neurocognitive Battery (CNB) (27). Accuracy of neurocognitive performance was measured across all tests included in the CNB, with raw measures of accuracy being normalized across the entire PNC. Executive function (EF) was summarized using a previously published factor analysis of the CNB (28). Each participant's $z$-score from this factor analysis was used to evaluate associations between executive function and CBF-ALFF coupling.

\section{Image acquisition}

PNC imaging was acquired at a single site with a $3 \mathrm{~T}$ Siemens Tim Trio scanner with a 32-channel head coil (Erlangen, Germany), as previously described (22). To minimize motion, prior to data acquisition, subjects' heads were stabilized in the head coil using one foam pad over each ear and a third over the top of the head.

High-resolution structural images were acquired to facilitate alignment of individual subject images into a common space. Structural images were acquired using a 3D-encoded magnetization-prepared, rapid-acquisition gradient-echo (MPRAGE) T1-weighted sequence $\left(\mathrm{T}_{\mathrm{R}}=1810 \mathrm{~ms} ; \mathrm{T}_{\mathrm{E}}=3.51 \mathrm{~ms} ; \mathrm{FoV}=180 \times 240 \mathrm{~mm}\right.$; matrix size $=192 \times 256$, number of slices $=$ 160, slice thickness $/$ gap $=1 \mathrm{~mm} / 0$; resolution $0.9375 \times 0.9375 \times 1 \mathrm{~mm}$ ).

Approximately 6 minutes of task-free functional data were acquired for each subject using a blood oxygen level-dependent (BOLD-weighted) 2D EPI sequence $\left(\mathrm{T}_{\mathrm{R}}=3000 \mathrm{~ms}\right.$; $\mathrm{T}_{\mathrm{E}}=32 \mathrm{~ms} ; \mathrm{FoV}=192 \times 192 \mathrm{~mm}$; matrix size $=64 \times 64$; number of slices $=46$; slice thickness $/$ gap $=3 \mathrm{~mm} / 0$; resolution $3 \mathrm{~mm}$ isotropic; 124 volumes). A fixation cross was displayed as images were acquired. Subjects were instructed to stay awake, keep their eyes open, fixate on 
the displayed crosshair, and remain still. Brain perfusion was imaged with a 3D-encoded spinecho pseudocontinuous arterial spin labeling $(\mathrm{pCASL})$ sequence $\left(\mathrm{T}_{\mathrm{R}}=4000 \mathrm{~ms} ; \mathrm{T}_{\mathrm{E}}=15 \mathrm{~ms}\right.$; FoV $=220 \times 220 \mathrm{~mm}$; matrix size $=96 \times 96$; number of slice $=20 ;$ slice thickness $/$ gap $=5 / 1 \mathrm{~mm}$; resolution $2.3 \times 2.3 \times 6 \mathrm{~mm}$; 80 volumes).

\section{Statistical Analyses}

Image processing

The structural images were processed using FreeSurfer (version 5.3) to allow for the projection of functional timeseries to the cortical surface (29). Resting-state fMRI scans were processed using a top-performing preprocessing pipeline implemented using the eXtensible Connectivity Pipelines (XCP) (30), which includes tools from FSL $(31,32)$ and AFNI (33). This pipeline included (1) correction for distortions induced by magnetic field inhomogeneity using FSL's FUGUE utility, (2) removal of the initial 4 volumes for resting-state fMRI, (3) realignment of all volumes to a selected reference volume using FSL's MCFLIRT, (4) interpolation of intensity outliers in each voxel's time series using AFNI's 3dDespike utility, (5) demeaning and removal of any linear or quadratic trends, and (6) co-registration of functional data to the high-resolution structural image using boundary-based registration. Images were denoised using a 36-parameter confound regression model that has been shown to minimize associations with motion artifact while retaining signals of interest in distinct sub-networks (30). This model included the six framewise estimates of motion, the mean signal extracted from eroded white matter and cerebrospinal fluid compartments, the mean signal extracted from the entire brain, the derivatives of each of these nine parameters, and quadratic terms of each of the nine parameters and their derivatives. Both the BOLD-weighted time series and the artifactual 
model time series were temporally filtered using a first-order Butterworth filter with a passband between 0.01 and $0.08 \mathrm{~Hz}$ to avoid mismatch in the temporal domain (34). The voxel-wise ALFF was computed as the sum over frequency bins in the low-frequency $(0.01-0.08$ Hertz $)$ band of the power spectrum using a Fourier transform of the time-domain signal (14).

As part of image quality assurance, T1-weighted images were excluded for low quality and/or low quality in FreeSurfer reconstruction, as rated by three independent reviewers (35). ASL images were excluded if they had excessive motion (mean relative displacement $>0.5 \mathrm{~mm}$ ), low temporal signal-to-noise ratio $(\mathrm{tSNR}<30)$, poor image coverage, or an excessive number of voxels that had ceiling intensity values at some point in the timeseries $(36,37)$. Task-free BOLD scans were excluded if the mean relative root mean square (RMS) framewise displacement was higher than $0.2 \mathrm{~mm}$, or if there were more than 20 frames with motion exceeding $0.25 \mathrm{~mm}(22)$.

To obtain CBF maps from ASL MRI, images were also processed with tools from XCP (38). CBF was quantified from control-label pairs in the following equation:

$$
f=\frac{\Delta M \lambda R_{1 a} \exp \left(\omega R_{1 a}\right)}{2 M_{0} \alpha}\left[1-\exp \left(-\tau R_{1 a}\right)\right]^{-1}
$$

where $f$ is $\mathrm{CBF}, \Delta M$ is the difference signal between the control and label acquisitions, $R_{1 a}$ is the longitudinal relaxation rate of blood, $\tau$ is the labeling time, $\omega$ is the postlabeling delay time, $\alpha$ is the labeling efficiency, $\lambda$ is the blood/tissue water partition coefficient, and $M_{0}$ is approximated by the control image intensity (38). We set $\alpha=0.85, \lambda=0.9 \mathrm{~g} / \mathrm{mL}, \tau=1.6 \mathrm{~s}$, and $\omega=1.2 \mathrm{~s}$. Participant-level CBF images were co-registered to the corresponding T1-weighted image using boundary-based registration with six degrees of freedom (39). Given that T1 relaxation time differs according to age and sex (40-42), the T1 relaxation parameter was modeled on an age- 
and sex-specific basis (43). This has been shown to enhance the accuracy and reliability of results in developmental samples $(36,44)$.

The CBF and ALFF maps for each individual were projected to the participant's anatomic surface and smoothed with a $6 \mathrm{~mm}$ full-width half-maximum (FWHM) kernel. The

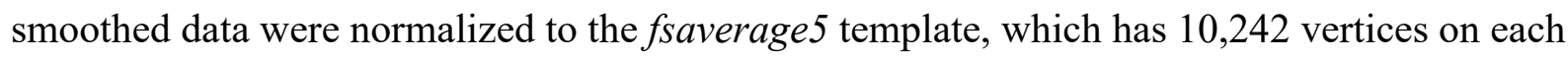
hemisphere (18,715 vertices in total after removing the medial wall).

\section{$C B F-A L F F$ coupling}

Coupling maps were generated at the cortical surface using methods as previously described in detail (24). For each vertex, a 15-mm FWHM neighborhood was defined and a locally weighted regression where ALFF was predicted by CBF was fit. This was repeated at all vertices within each subject, generating one CBF-ALFF coupling map where each vertex was represented by the coupling regression slope (Figure 2). A group mean coupling map was also generated by averaging the mean $t$-statistic of each individual's CBF-ALFF slope at a given vertex. 
A

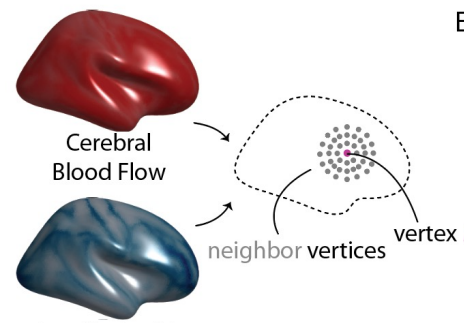

Amplitude of Low Frequency Fluctuations

D

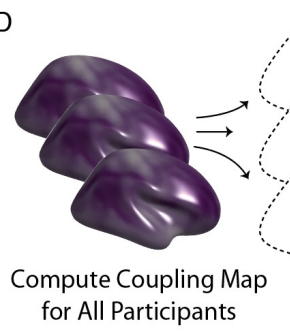

B Locally Weighted Linear Regression

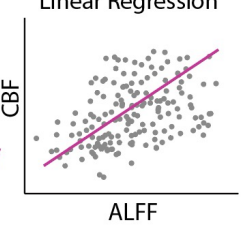

ALFF

E

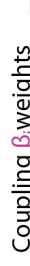

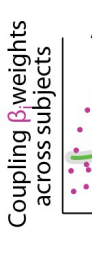

Generalized Aditive Models $\therefore 0^{\circ} \because 0^{\circ}$ $\because \div \cdots$ $\because \because \therefore \because$ Age, Sex, or
Exec Function
C

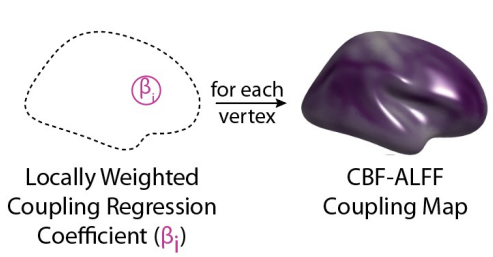

F

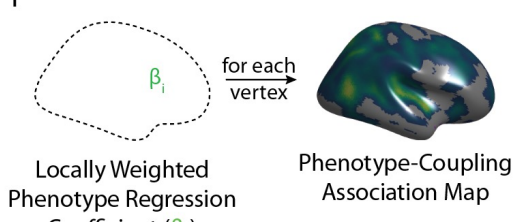

Figure 2. Analysis of CBF-ALFF coupling. CBF-ALFF coupling analysis involves both calculation of within-subject coupling and across-subject comparisons to assess individual differences. A) For each subject, a neighborhood for each vertex was identified. B) Locally weighted regressions of ALFF onto CBF were calculated. C) Locally weighted regressions were repeated at each vertex, resulting in a participant-level coupling map. D-E) After subject-level coupling maps were calculated, statistical analyses related covariates of interest (e.g., Age, Sex, and Executive Function) to participant-level coupling maps using generalized additive models (GAMs). F) GAMs were fit at each vertex, yielding a group-level statistical map describing individual differences.

\section{Statistical analyses and hypothesis testing}

We sought to evaluate how neurovascular coupling develops and relates to biological sex and executive function. CBF-ALFF coupling maps for each participant were used for statistical analyses. Generalized additive models (GAMs) were used to calculate linear and nonlinear age and sex effects at each vertex using the following model:

Coupling $_{\text {vertex }}=$ spline $($ age $)+$ sex + CBF motion + ALFF motion + error

This approach allowed for flexible modeling of both linear and nonlinear effects. For significance testing, smooth terms were fitted as fixed degrees of freedom regression splines $(\mathrm{k}=$ 4). We also fit the model with an age-by-sex interaction; however, it was not significant and thus removed from the model. To assess the relationship between coupling and executive function 
accuracy, we fit a second model which included a linear executive accuracy term in addition to the above model's covariates (spline of age, sex, CBF and ALFF motion, and error). GAMs were estimated using the $\mathbf{R}$ package 'mgcv' in CRAN (45). All analyses controlled the False Discovery Rate (FDR) at $Q<0.05$.

In addition to these analyses of effects at each vertex, we also evaluated the relationship between whole-brain coupling and age nonlinearly. We first computed a single mean t-statistic per subject by averaging the t-statistics for the slope at each vertex that met FDR correction, and then used a GAM to estimate the relationship between each subject's mean coupling and age. To test for windows of significant change across the age range, we calculated the first derivative of the smooth function of age from the GAM model using finite differences, and then generated a simultaneous $95 \%$ confidence interval of the derivative using the $\mathbf{R}$ package 'gratia` (45-47). Intervals of significant change were identified as areas where the simultaneous confidence interval of the derivative did not include zero.

\section{Network enrichment analyses via spin testing}

Given that patterns of neural activity differ across functional networks (48), we sought to characterize whether effects of interest tended to be located within specific functional brain networks $(49,50)$. To do this, we conducted network enrichment analyses. Specifically, we evaluated whether mean coupling and significant associations with variables of interest (age, sex, executive function) were preferentially located in one or more of the seven large-scale functional networks defined by Yeo et al (49). To account for the different size of each network and the spatial autocorrelation of brain maps, statistical testing used a conservative spin-based spatial permutation procedure (51). Briefly, statistical maps from association testing were projected onto 
a sphere, which was rotated 1,000 times per hemisphere to create a null distribution. For the mean coupling enrichment analysis, the test statistic was the mean $t$-value for each of the seven networks from Yeo et al. For the regression analyses, the test statistic was the proportion of vertices that survived FDR correction. Networks were considered to have significant enrichment if the test statistic in the observed data was in the top 5\% of the null distribution derived from permuted data.

\section{Code availability}

Code for all primary statistical analyses is available at:

https://github.com/PennLINC/IntermodalCoupling

\section{RESULTS}

\section{$C B F$ and ALFF are significantly coupled}

Replicating previous findings in adults, we observed robust CBF-ALFF coupling throughout the brain (Figure 3A). Coupling was strongest in association cortices, including frontal, parietal, and temporal cortex $\left(p_{f d r}<0.05\right)$. Network enrichment analysis using spin-based permutation tests revealed enrichment of CBF-ALFF coupling in the default mode network $(p=$ 0.045), with nominally higher coupling in the frontoparietal network ( $p=0.056$; Figure 3B). 

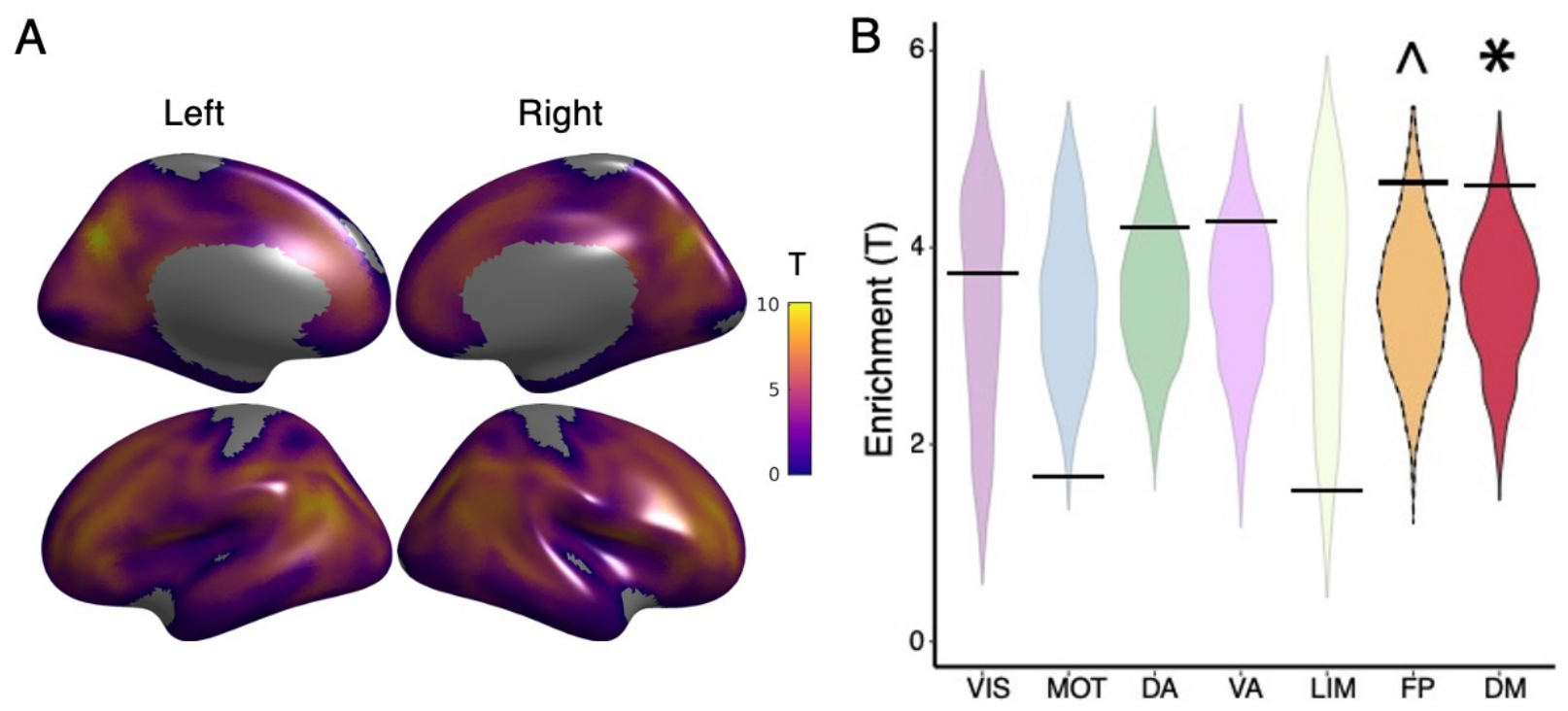

Figure 3. Mean CBF-ALFF coupling. A) CBF-ALFF coupling is robust throughout the brain, with maximal coupling in the medial and lateral prefrontal cortex, parietal cortex, posterior cingulate and precuneus. B) We used a spin-based spatial permutation test that accounted for spatial autocorrelation to evaluate enrichment of CBF-ALFF coupling in canonical functional networks. This revealed enrichment in the default mode network $(p=0.045)$ with a trend in the frontoparietal network $(p=0.056)$. Star $(*)$ represents statistical significance $(p<0.05)$ and caret $\left({ }^{\wedge}\right)$ represents a non-significant trend $(p<0.1)$. The black bars represent the observed values, whereas the violin plots reflect the null distributions.

\section{CBF-ALFF coupling declines with adolescent development}

Next, we evaluated associations between CBF-ALFF coupling and age. We utilized a generalized additive model to rigorously evaluate both linear and nonlinear developmental effects, while controlling for in-scanner motion and sex. We found that whole-brain mean coupling decreased across development $\left(\mathrm{F}_{3,828}=60.0, p<0.0001\right.$; Figure 4A). Analysis of the derivative of this spline revealed that coupling significantly decreased between 11.6 years and 20.5 years of age, with a peak decline observed during mid-adolescence at age 16 . Fitting this model at each vertex revealed widespread declines in CBF-ALFF coupling across much of the cortex, with peak effects present in the posterior temporal cortex $\left(p_{f d r}<0.05\right.$; Figure 4B).

Analyses using spin-based permutation testing revealed enrichment of age-related declines in 
coupling within the dorsal attention network $(p=0.014$; Figure 4C). In contrast to these spatially extensive declines in coupling, only small regions in bilateral temporal cortices (75 vertices total) showed increased coupling with age.
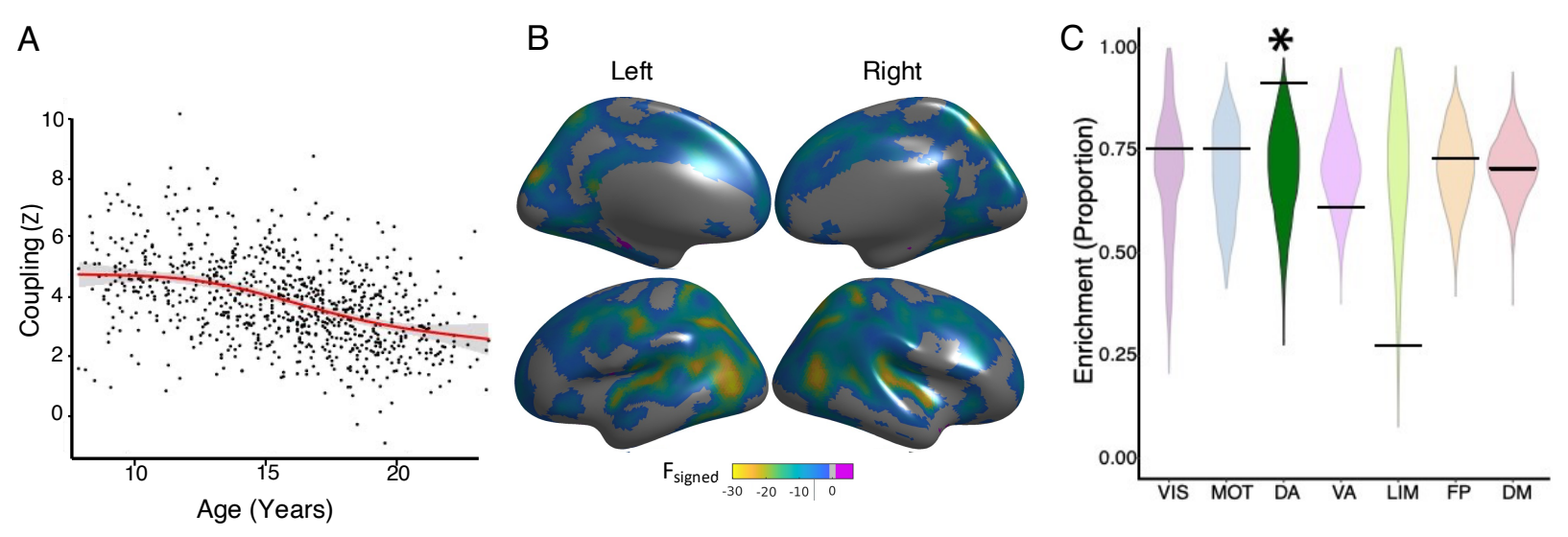

Figure 4. CBF-ALFF coupling evolves with age. Linear and non-linear age effects of CBFALFF were flexibly modeled within a generalized additive model at each vertex, while controlling for sex and in-scanner motion; multiple comparisons were controlled using the False Discovery Rate $(Q<0.05)$. A) Mean cortical CBF-ALFF coupling declines with age in a nonlinear fashion $\left(\mathrm{F}_{3,282}=60.0, p<0.0001\right)$. Data points represent the mean CBF-ALFF coupling $(Z)$ for each subject $(\mathrm{n}=831)$ across all vertices that met statistical correction $\left(p_{f d r}<0.05\right)$. B) Vertex-level CBF-ALFF declines were prominent in the posterior temporal cortex, parietal cortex, and dorsolateral prefrontal cortex. For visualization purposes, $\mathrm{F}_{\text {signed }}$ refers to the F-value from the generalized additive models, with the sign representing directionality (e.g., negative numbers represent lower coupling with age). C) Spin testing revealed enrichment of age effects within the dorsal attention network $(p=0.014)$. Star $(*)$ represents statistical significance. Black bars represent the observed values, whereas the violin plots reflect the null distributions.

\section{CBF-ALFF coupling is higher in females within the frontoparietal network}

Having established significant declines in CBF-ALFF coupling with age, we next evaluated sex differences while controlling for both linear and nonlinear effects of age (as well as in-scanner motion). We found that females had stronger coupling than males in bilateral dorsolateral prefrontal cortex, medial frontal cortex, anterior cingulate cortex, and precuneus. In contrast, females had lower coupling in the cuneus and lateral temporal cortex $\left(p_{f d r}<0.05\right.$; 
Figure 5A). Spin testing revealed that sex differences in coupling were enriched within the frontoparietal network $(p=0.034$; Figure 5B).
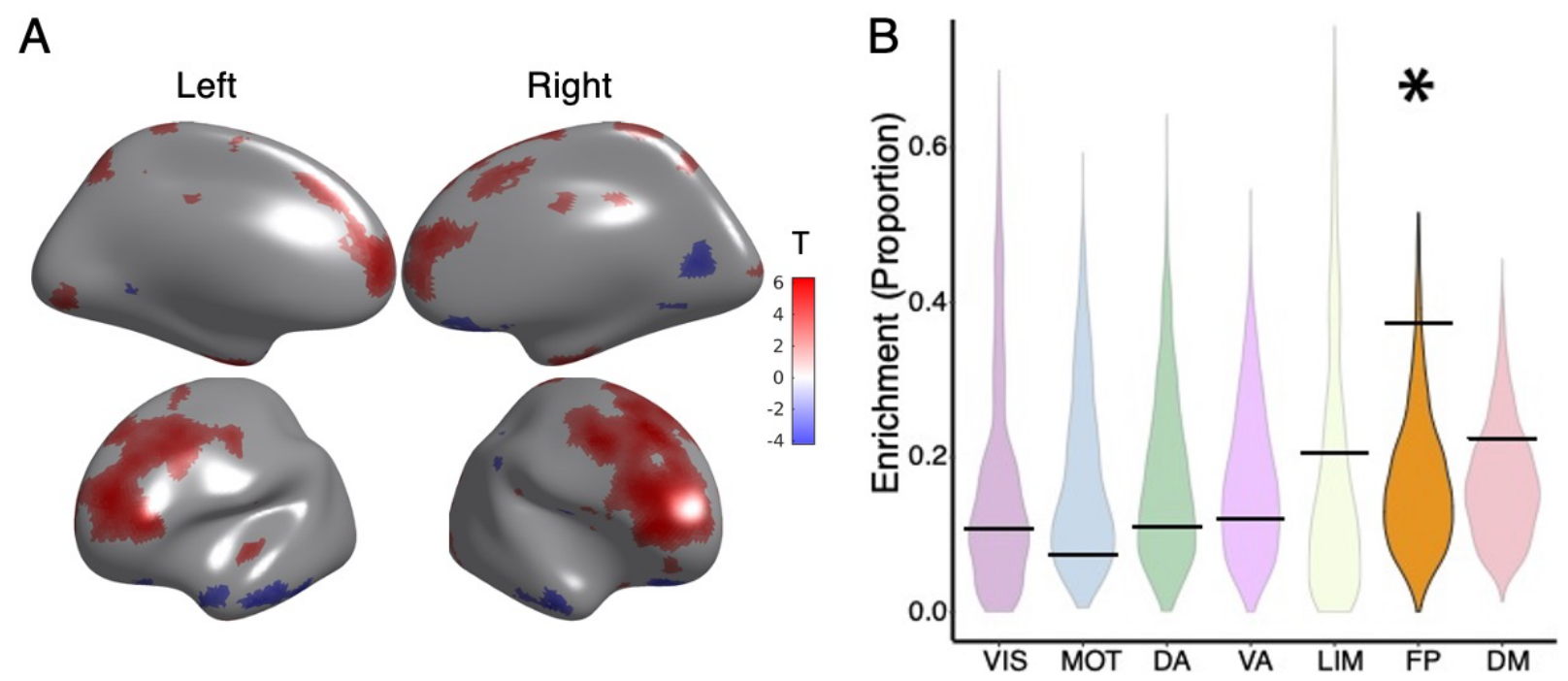

Figure 5. Sex differences in CBF-ALFF coupling. A) CBF-ALFF coupling is higher in females than males in the bilateral dorsolateral prefrontal cortex, medial frontal cortex, anterior cingulate cortex, and precuneus. CBF-ALFF coupling differences between females and males were modeled using generalized additive models, while adjusting for both linear and nonlinear age effects as well as in-scanner motion; multiple comparisons were controlled using the False Discovery Rate $(Q<0.05)$. Brain regions where females had greater coupling than males are shown in red, whereas areas where males have greater coupling than females are shown in blue. B) Spin testing revealed significant enrichment of sex differences within the frontoparietal network $(p=0.034)$. Star $\left(^{*}\right)$ represents statistical significance. Black bars represent the observed values, whereas the violin plots reflect the null distributions.

\section{CBF-ALFF coupling is associated with executive function}

As a final step, we evaluated the relationship between CBF-ALFF coupling and executive function. Throughout, we controlled for linear and nonlinear age effects, sex, and in-scanner motion. We found that better executive function was related to higher coupling in default mode regions including the posterior cingulate cortex, medial prefrontal cortex, and left temporoparietal junction. Furthermore, lower executive function was also related to more coupling in bilateral motor cortex and primary auditory cortex, including Heschl's gyrus $\left(p_{f d r}<\right.$ 
0.05; Figure 6A). Spin testing revealed enrichment of significant associations with executive function within the somatomotor network $(p=0.040$; Figure 6B).
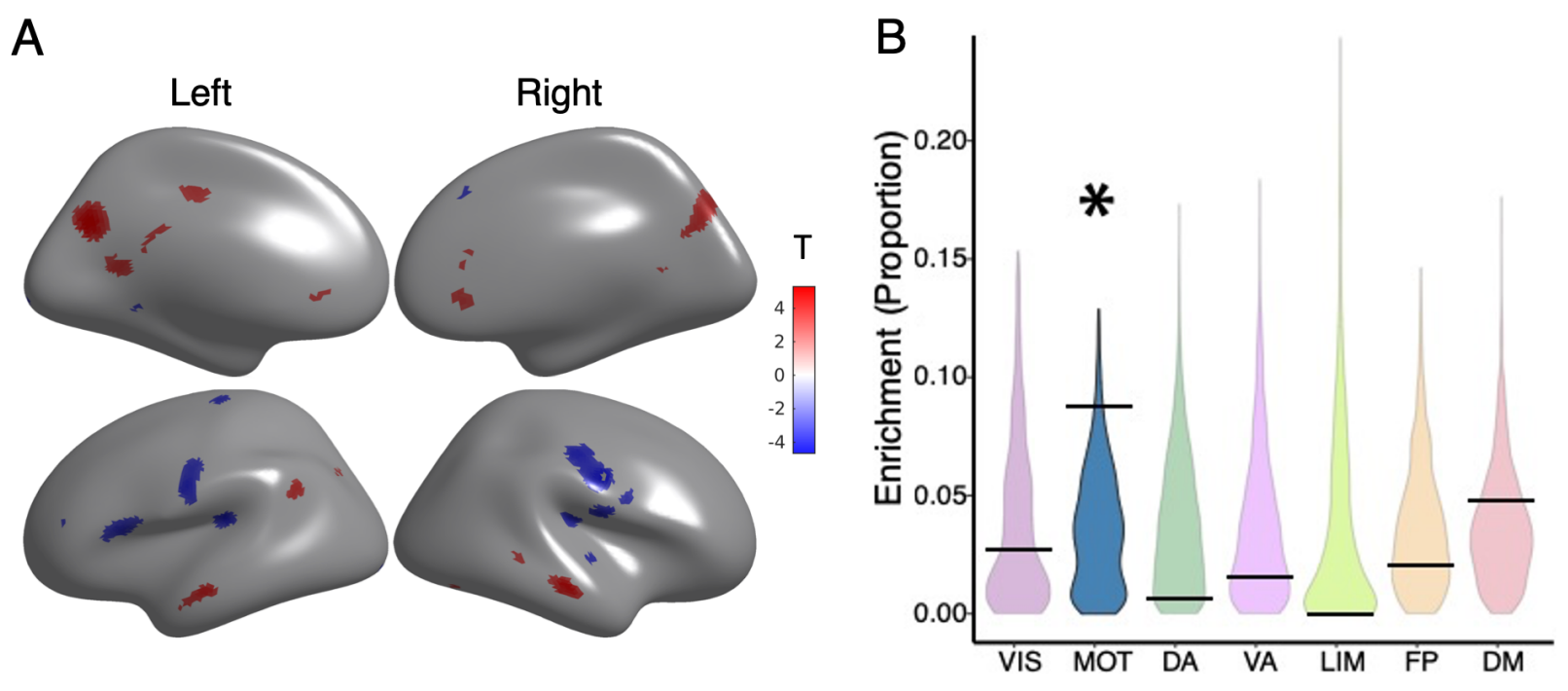

Figure 6. CBF-ALFF coupling is related to executive function. A) The relationship of CBFALFF coupling to executive function showed regional variation, with both positive and negative associations. Generalized additive models were used to calculate the relationship between CBFALFF coupling and executive function while controlling for linear and nonlinear age effects, sex effects, and in-scanner motion; multiple comparisons were accounted for using the False Discovery Rate $(Q<0.05)$. Higher coupling in parts of the default mode were associated with better executive functioning, while higher coupling in parts of the somatomotor network were associated with reduced executive functioning. Brain regions with positive associations are shown in red, whereas areas with negative associations are shown in blue. B) Spin testing revealed that associations between executive function and coupling were significantly enriched in the motor network $(p=0.040)$. Star $(*)$ represents statistical significance $(p<0.05)$. Black bars represent the observed values, whereas the violin plots reflect the null distributions.

\section{DISCUSSION}

Using a recently developed statistical method for assessing inter-modal coupling and a large sample of youth, we demonstrated significant CBF-ALFF coupling across the cortex. Furthermore, we identified age-related declines in coupling that were broadly distributed across cortex and were enriched in the dorsal attention network. In addition, we found that sex differences in CBF-ALFF coupling were enriched within the frontoparietal network. Finally, we highlighted the relevance of CBF-ALFF coupling for cognition by showing significant 
associations between CBF-ALFF with executive function. Taken together, these results extend prior results in adults and demonstrate that CBF-ALFF coupling undergoes a process of developmental calibration that is relevant for cognition.

Neurovascular coupling is thought to reflect the interrelationship between nutrient demand and supply, whereby neuronal activity influences local changes in blood flow $(6,7,21,52)$. At the cellular level, researchers have demonstrated a close relationship between blood flow and neural function that is usually facilitated by communicating astrocytes, where ionic gradients and metabolic biproducts from firing neurons lead to local vasodilation of cerebral arterioles $(53,54)$. As such, high coupling could be conceptualized as an optimized, high fidelity system, where local activity of the vascular unit is influenced by neuronal function (55). Prior work has suggested that CBF-ALFF coupling may be understood as a proxy of neurovascular coupling, allowing a non-invasive window into this process $(9,18,19)$.

Highly metabolically active regions, particularly in association networks that coordinate brain activity across distributed brain regions, tend to have increased CBF as well as ALFF $(16,56)$. Here, we showed that CBF-ALFF coupling is enriched in the default mode network $(\mathrm{DMN})$, with a trend toward significance in the frontoparietal network (FPN). Transmodal association cortices that subserve the DMN and FPN have larger pyramidal neurons with greater spine and synapse density than pyramidal neurons expressed in other parts of the brain (57-59). Similarly, these association networks are the most spatially distributed, with more long-distance cortico-cortical connections (60-63). These neuroanatomical features produce greater metabolic activity and thus may demand a tighter link between activity and blood flow (64).

Though coupling remained tight across development and into young adulthood, CBF-ALFF coupling decreased across much of the cortex, with the greatest rate of change during mid- 
adolescence. These findings coincide with many previously described structural developmental brain changes (65-67). During the second decade of life, myelin increases while synapses and dendritic spines are pruned to facilitate more efficient between-neuron communication (68-71). To support these structural changes, perineuronal nets surround the cell bodies and dendrites and control ion flow and conduction (72). One possibility is that these structural and ionic adaptations reduce the metabolic demands of the neurons, which has been previously demonstrated in perfusion studies $(36,73)$. These refined local neural circuits may allow for fluctuations in neural activity to be supported by a lower level of metabolic substrate delivered by blood flow.

Sex differences in coupling followed a different pattern than age-related changes. Females showed significantly higher coupling in the FPN, which is essential for cognitive control (74). Interestingly, previous literature has demonstrated that prominent sex differences in perfusion also emerge during the same developmental window, when girls experience an increase in circulating ovarian estrogens $(36,37,75)$. The neuroactive steroid $17 \mathrm{~B}$ estradiol, the primary estrogen secreted from the ovary, functions as a potent neurovasodilator by enhancing the production of nitric oxide (76). Neurophysiologic studies have consistently demonstrated larger cerebral blood vessel diameter per unit blood pressure in females as compared to males $(77,78)$. Estrogen is also known to selectively increase blood flow in executive areas in adults (79) and emerging evidence has established a link between higher estradiol levels and greater dorsolateral prefrontal cortex activity during emotion regulation in adolescents (80). It is possible that hormone-mediated mechanisms contribute to observed sex differences in coupling within the FPN. 
Understanding sex differences in frontoparietal neurobiology that emerge in adolescence is critically important given that mood and anxiety disorders, which are twice as prevalent in girls than boys, also emerge during the same developmental window $(81,82)$. The adult literature consistently reports altered FPN functioning in depression and anxiety $(83,84)$, and these differences can also be identified in development. Previous fMRI research has linked variations in frontoparietal network function with phenotypic heterogeneity in youth depression (85) and anxiety disorders (86). As a next step, it will be important to study whether frontoparietal coupling could be used as a biomarker for sex differences in psychopathology.

The relevance of CBF-ALFF coupling to cognitive function is supported by our results, which detail a significant age-independent relationship between CBF-ALFF coupling and executive function. Specifically, better executive function was associated with lower coupling in the somatomotor network and higher coupling in regions within the DMN, including the posterior cingulate cortex and medial prefrontal cortex. Our findings align with previous unimodal neuroimaging literature on cognition in youth, where executive functioning has been related to both lower-order sensorimotor networks as well as higher-order association networks $(87,88)$. Some previous studies have also suggested a dissociation between how executive functioning relates to neuroimaging measures of sensorimotor and association networks (89). Our findings may further indicate that the development of executive function in youth relies on a balance between decreased coupling in lower-order networks, and increased coupling in higherorder association networks. It is also possible that this dissociation is specific to the adolescent developmental stage, where lower coupling in somatomotor network indicates a refined circuit, whereas higher correlation in the DMN indicates that the system is undergoing developmental tuning and would presumably move to a lower coupling state in adulthood. 
There are several limitations to our study which should be noted. Previous authors have understood CBF-ALFF coupling as a representation of neurovascular coupling, which has framed our understanding of this measure $(12,18,19)$. However, we are not able to measure neurovascular coupling directly and had to rely on proxy measures to characterize neurovascular coupling in vivo. Typically, positron emission tomography has generally been the gold standard for measuring blood flow, whereas we use ASL to quantify CBF. However, previous studies have demonstrated good correlation between PET and ASL (13). Given the potential risks of radiologic exposure to children with PET scanning, CBF as measured by ASL is a much safer method for use in large-scale studies of youth (90-92). Additionally it should be noted that ALFF is derived from the BOLD signal, which inherently has a vascular component (93). However, numerous past studies have suggested that ALFF signals reflect neural activity $(14,15,94,95)$. Lastly, we evaluated a cross-sectional sample which prevents us from estimating within-individual change; future studies in longitudinal samples will be important.

The limitations notwithstanding, we provide new evidence for the developmental evolution of CBF-ALFF coupling in youth, as well as distinct associations with sex and executive function. Our findings suggest numerous avenues for future study. Longitudinal assessments that allow for the measure of within-subject change in coupling may help us better understand normal physiologic brain development at a personalized level. Additionally, transdiagnostic deficits in executive functioning are commonly observed across psychiatric illnesses $(96,97)$. Given that deficits in executive functioning were associated with altered CBF-ALFF coupling, future studies could yield important insights into whether regional differences in CBF-ALFF coupling are linked to the onset and development of psychopathology. Eventually, characterizing 
neurovascular coupling in youth at risk may aid in the development of targeted pharmacologic and neurotherapeutic treatments.

\section{REFERENCES}

1. Kety, Seymour. The general metabolism of the brain in vivo. In: Metabolism of the nervous system. London: Pergamon; 1957. p. 221-37.

2. Sokoloff L. Cerebral Metabolism and Visualization of Cerebral Activity. In: Greger R, Windhorst U, editors. Comprehensive Human Physiology: From Cellular Mechanisms to Integration [Internet]. Berlin, Heidelberg: Springer; 1996 [cited 2021 Jun 25]. p. 579-602. Available from: https://doi.org/10.1007/978-3-642-60946-6_30

3. Attwell D, Laughlin SB. An Energy Budget for Signaling in the Grey Matter of the Brain. J Cereb Blood Flow Metab. 2001 Oct 1;21(10):1133-45.

4. Xing C-Y, Tarumi T, Liu J, Zhang Y, Turner M, Riley J, et al. Distribution of cardiac output to the brain across the adult lifespan. J Cereb Blood Flow Metab. 2017 Aug;37(8):2848-56.

5. Williams LR, Leggett RW. Reference values for resting blood flow to organs of man. Clin Phys Physiol Meas. 1989 Aug;10(3):187-217.

6. Armstead WM. Cerebral Blood Flow Autoregulation and Dysautoregulation. Anesthesiol Clin. 2016 Sep;34(3):465-77.

7. Kim KJ, Filosa JA. Advanced in vitro approach to study neurovascular coupling mechanisms in the brain microcirculation. J Physiol. 2012 Apr 1;590(7):1757-70.

8. Phillips AA, Chan FH, Zheng MMZ, Krassioukov AV, Ainslie PN. Neurovascular coupling in humans: Physiology, methodological advances and clinical implications. J Cereb Blood Flow Metab. 2016 Apr;36(4):647-64.

9. Girouard H, Iadecola C. Neurovascular coupling in the normal brain and in hypertension, stroke, and Alzheimer disease. J Appl Physiol (1985). 2006 Jan;100(1):328-35.

10. Garrett DD, Lindenberger U, Hoge RD, Gauthier CJ. Age differences in brain signal variability are robust to multiple vascular controls. Scientific Reports. 2017 Aug 31;7(1):10149.

11. Li Z, Zhu Y, Childress AR, Detre JA, Wang Z. Relations between BOLD fMRI-Derived Resting Brain Activity and Cerebral Blood Flow. PLOS ONE. 2012 Sep 21;7(9):e44556.

12. Zheng W, Cui B, Han Y, Song H, Li K, He Y, et al. Disrupted Regional Cerebral Blood Flow, Functional Activity and Connectivity in Alzheimer's Disease: A Combined ASL 
Perfusion and Resting State fMRI Study. Front Neurosci [Internet]. 2019 Jul 24 [cited 2021 Apr 9];13. Available from: https://www.ncbi.nlm.nih.gov/pmc/articles/PMC6668217/

13. Detre JA, Alsop DC. Perfusion magnetic resonance imaging with continuous arterial spin labeling: methods and clinical applications in the central nervous system. European Journal of Radiology. 1999 May;30(2):115-24.

14. Cordes D, Haughton VM, Arfanakis K, Carew JD, Turski PA, Moritz CH, et al. Frequencies contributing to functional connectivity in the cerebral cortex in "resting-state" data. AJNR Am J Neuroradiol. 2001 Aug;22(7):1326-33.

15. Biswal B, Yetkin FZ, Haughton VM, Hyde JS. Functional connectivity in the motor cortex of resting human brain using echo-planar MRI. Magn Reson Med. 1995 Oct;34(4):537-41.

16. Zou Q-H, Zhu C-Z, Yang Y, Zuo X-N, Long X-Y, Cao Q-J, et al. An improved approach to detection of amplitude of low-frequency fluctuation (ALFF) for resting-state fMRI: Fractional ALFF. J Neurosci Methods. 2008 Jul 15;172(1):137-41.

17. Ma X, Wang D, Zhou Y, Zhuo C, Qin W, Zhu J, et al. Sex-dependent alterations in restingstate cerebral blood flow, amplitude of low-frequency fluctuations and their coupling relationship in schizophrenia. Aust N Z J Psychiatry. 2016 Apr;50(4):334-44.

18. Hu B, Yan L-F, Sun Q, Yu Y, Zhang J, Dai Y-J, et al. Disturbed neurovascular coupling in type 2 diabetes mellitus patients: Evidence from a comprehensive fMRI analysis.

Neuroimage Clin [Internet]. 2019 Mar 27 [cited 2020 Nov 7];22. Available from: https://www.ncbi.nlm.nih.gov/pmc/articles/PMC6447740/

19. Jin M, Wang L, Wang H, Han X, Diao Z, Guo W, et al. Disturbed neurovascular coupling in hemodialysis patients. PeerJ [Internet]. 2020 Apr 15 [cited 2020 Nov 7];8. Available from: https://www.ncbi.nlm.nih.gov/pmc/articles/PMC7166048/

20. Gur RC, Butler ER, Moore TM, Rosen AFG, Ruparel K, Satterthwaite TD, et al. Structural and Functional Brain Parameters Related to Cognitive Performance Across Development: Replication and Extension of the Parieto-Frontal Integration Theory in a Single Sample. Cereb Cortex. 2021 Feb 5;31(3):1444-63.

21. Presa JL, Saravia F, Bagi Z, Filosa JA. Vasculo-Neuronal Coupling and Neurovascular Coupling at the Neurovascular Unit: Impact of Hypertension. Front Physiol [Internet]. 2020 [cited 2021 May 14];11. Available from: https://www.frontiersin.org/articles/10.3389/fphys.2020.584135/full

22. Satterthwaite TD, Elliott MA, Ruparel K, Loughead J, Prabhakaran K, Calkins ME, et al. Neuroimaging of the Philadelphia Neurodevelopmental Cohort. Neuroimage. 2014;86:54453.

23. Satterthwaite TD, Connolly JJ, Ruparel K, Calkins ME, Jackson C, Elliott MA, et al. The Philadelphia Neurodevelopmental Cohort: a publicly available resource for the study of normal and abnormal brain development in youth. Neuroimage. 2016;124:1115-9. 
24. Vandekar SN, Shinohara RT, Raznahan A, Hopson RD, Roalf DR, Ruparel K, et al. Subject-level measurement of local cortical coupling. NeuroImage. 2016 Jun;133:88-97.

25. Vandekar SN, Shinohara RT, Raznahan A, Roalf DR, Ross M, DeLeo N, et al. Topologically Dissociable Patterns of Development of the Human Cerebral Cortex. The Journal of Neuroscience. 2015 Jan 14;35(2):599-609.

26. Calkins ME, Merikangas KR, Moore TM, Burstein M, Behr MA, Satterthwaite TD, et al. The Philadelphia Neurodevelopmental Cohort: constructing a deep phenotyping collaborative. Journal of Child Psychology and Psychiatry. 2015;56(12):1356-69.

27. Gur RC, Richard J, Calkins ME, Chiavacci R, Hansen JA, Bilker WB, et al. Age group and sex differences in performance on a computerized neurocognitive battery in children age 821. Neuropsychology. 2012 Mar;26(2):251-65.

28. Moore TM, Reise SP, Gur RE, Hakonarson H, Gur RC. Psychometric properties of the Penn Computerized Neurocognitive Battery. Neuropsychology. 2015;29(2):235.

29. Fischl B. FreeSurfer. Neuroimage. 2012 Aug 15;62(2):774-81.

30. Ciric R, Rosen AFG, Erus G, Cieslak M, Adebimpe A, Cook PA, et al. Mitigating head motion artifact in functional connectivity MRI. Nat Protoc. 2018 Dec;13(12):2801-26.

31. Jenkinson M, Beckmann CF, Behrens TEJ, Woolrich MW, Smith SM. FSL. NeuroImage. 2012 Aug 15;62(2):782-90.

32. Smith SM, Jenkinson M, Woolrich MW, Beckmann CF, Behrens TEJ, Johansen-Berg H, et al. Advances in functional and structural MR image analysis and implementation as FSL. Neuroimage. 2004;23 Suppl 1:S208-219.

33. Cox RW. AFNI: software for analysis and visualization of functional magnetic resonance neuroimages. Comput Biomed Res. 1996 Jun;29(3):162-73.

34. Hallquist MN, Hwang K, Luna B. The nuisance of nuisance regression: Spectral misspecification in a common approach to resting-state fMRI preprocessing reintroduces noise and obscures functional connectivity. NeuroImage. 2013 Nov 15;82:208-25.

35. Rosen AF, Roalf DR, Ruparel K, Blake J, Seelaus K, Villa LP, et al. Quantitative assessment of structural image quality. Neuroimage. 2018;169:407-18.

36. Satterthwaite TD, Shinohara RT, Wolf DH, Hopson RD, Elliott MA, Vandekar SN, et al. Impact of puberty on the evolution of cerebral perfusion during adolescence. Proceedings of the National Academy of Sciences. 2014;111(23):8643-8.

37. Kaczkurkin AN, Moore TM, Ruparel K, Ciric R, Calkins ME, Shinohara RT, et al. Elevated amygdala perfusion mediates developmental sex differences in trait anxiety. Biol Psychiatry. 2016 15;80(10):775-85. 
38. Wang Z, Aguirre GK, Rao H, Wang J, Fernández-Seara MA, Childress AR, et al. Empirical optimization of ASL data analysis using an ASL data processing toolbox: ASLtbx. Magn Reson Imaging. 2008 Feb;26(2):261-9.

39. bbregister - Free Surfer Wiki [Internet]. [cited 2021 May 15]. Available from: https://surfer.nmr.mgh.harvard.edu/fswiki/bbregister

40. Biagi L, Abbruzzese A, Bianchi MC, Alsop DC, Guerra AD, Tosetti M. Age dependence of cerebral perfusion assessed by magnetic resonance continuous arterial spin labeling. Journal of Magnetic Resonance Imaging. 2007;25(4):696-702.

41. Hales PW, Kawadler JM, Aylett SE, Kirkham FJ, Clark CA. Arterial Spin Labeling Characterization of Cerebral Perfusion during Normal Maturation from Late Childhood into Adulthood: Normal 'Reference Range' Values and Their Use in Clinical Studies. J Cereb Blood Flow Metab. 2014 May 1;34(5):776-84.

42. Taki Y, Hashizume H, Sassa Y, Takeuchi H, Wu K, Asano M, et al. Gender differences in partial-volume corrected brain perfusion using brain MRI in healthy children. Neuroimage. 2011 Oct 1;58(3):709-15.

43. Wu W-C, Jain V, Li C, Giannetta M, Hurt H, Wehrli FW, et al. In vivo venous blood T1 measurement using inversion recovery true-FISP in children and adults. Magn Reson Med. 2010 Oct;64(4):1140-7.

44. Jain V, Duda J, Avants B, Giannetta M, Xie SX, Roberts T, et al. Longitudinal reproducibility and accuracy of pseudo-continuous arterial spin-labeled perfusion MR imaging in typically developing children. Radiology. 2012 May;263(2):527-36.

45. Wood S. mgcv: Mixed GAM Computation Vehicle with Automatic Smoothness Estimation [Internet]. 2021 [cited 2021 Jun 25]. Available from: https://CRAN.R-

project.org/package $=\mathrm{mgcv}$

46. Simpson GL, Singmann H. gratia: Graceful 'ggplot'-Based Graphics and Other Functions for GAMs Fitted Using “mgcv" [Internet]. 2021 [cited 2021 Jun 30]. Available from: https://CRAN.R-project.org/package=gratia

47. Larsen B, Bourque J, Moore TM, Adebimpe A, Calkins ME, Elliott MA, et al. Longitudinal Development of Brain Iron Is Linked to Cognition in Youth. J Neurosci. 2020 Feb 26;40(9):1810-8.

48. Raut RV, Snyder AZ, Raichle ME. Hierarchical dynamics as a macroscopic organizing principle of the human brain. PNAS. 2020 Aug 25;117(34):20890-7.

49. Thomas Yeo BT, Krienen FM, Sepulcre J, Sabuncu MR, Lashkari D, Hollinshead M, et al. The organization of the human cerebral cortex estimated by intrinsic functional connectivity. J Neurophysiol. 2011 Sep;106(3):1125-65. 
50. Buckner RL, Krienen FM. The evolution of distributed association networks in the human brain. Trends Cogn Sci. 2013 Dec;17(12):648-65.

51. Alexander-Bloch AF, Shou H, Liu S, Satterthwaite TD, Glahn DC, Shinohara RT, et al. On testing for spatial correspondence between maps of human brain structure and function. NeuroImage. 2018 Sep 1;178:540-51.

52. Silverman A, Petersen NH. Physiology, Cerebral Autoregulation. In: StatPearls [Internet]. Treasure Island (FL): StatPearls Publishing; 2021 [cited 2021 May 12]. Available from: http:/www.ncbi.nlm.nih.gov/books/NBK553183/

53. Blanco VM, Stern JE, Filosa JA. Tone-dependent vascular responses to astrocyte-derived signals. Am J Physiol Heart Circ Physiol. 2008 Jun;294(6):H2855-63.

54. Takano T, Tian G-F, Peng W, Lou N, Libionka W, Han X, et al. Astrocyte-mediated control of cerebral blood flow. Nat Neurosci. 2006 Feb;9(2):260-7.

55. Mulligan SJ, MacVicar BA. Calcium transients in astrocyte endfeet cause cerebrovascular constrictions. Nature. 2004 Sep 9;431(7005):195-9.

56. Passow S, Specht K, Adamsen TC, Biermann M, Brekke N, Craven AR, et al. Defaultmode network functional connectivity is closely related to metabolic activity. Hum Brain Mapp. 2015 Jun;36(6):2027-38.

57. Beul SF, Hilgetag CC. Neuron density fundamentally relates to architecture and connectivity of the primate cerebral cortex. Neuroimage. 2019 Apr 1;189:777-92.

58. Jacobs B, Schall M, Prather M, Kapler E, Driscoll L, Baca S, et al. Regional Dendritic and Spine Variation in Human Cerebral Cortex: a Quantitative Golgi Study. Cerebral Cortex. 2001 Jun 1;11(6):558-71.

59. Elston GN, Fujita I. Pyramidal cell development: postnatal spinogenesis, dendritic growth, axon growth, and electrophysiology. Front Neuroanat. 2014 Aug 12;8:78.

60. Jung J, Cloutman LL, Binney RJ, Lambon Ralph MA. The structural connectivity of higher order association cortices reflects human functional brain networks. Cortex. 2017 Dec;97:221-39.

61. Bassett DS, Bullmore E, Verchinski BA, Mattay VS, Weinberger DR, Meyer-Lindenberg A. Hierarchical Organization of Human Cortical Networks in Health and Schizophrenia. J Neurosci. 2008 Sep 10;28(37):9239-48.

62. Sepulcre J, Liu H, Talukdar T, Martincorena I, Yeo BTT, Buckner RL. The Organization of Local and Distant Functional Connectivity in the Human Brain. PLOS Computational Biology. 2010 Jun 10;6(6):e1000808.

63. Bazinet V, Wael RV de, Hagmann P, Bernhardt BC, Misic B. Multiscale communication in cortico-cortical networks. bioRxiv. 2020 Oct 2;2020.10.02.323030. 
64. Shokri-Kojori E, Tomasi D, Alipanahi B, Wiers CE, Wang G-J, Volkow ND. Correspondence between cerebral glucose metabolism and BOLD reveals relative power and cost in human brain. Nat Commun. 2019 Feb 11;10(1):690.

65. Giedd JN, Blumenthal J, Jeffries NO, Castellanos FX, Liu H, Zijdenbos A, et al. Brain development during childhood and adolescence: a longitudinal MRI study. Nature neuroscience. 1999;2(10):861.

66. Baum GL, Cui Z, Roalf DR, Ciric R, Betzel RF, Larsen B, et al. Development of structurefunction coupling in human brain networks during youth. PNAS. 2020 Jan 7;117(1):771-8.

67. Vázquez-Rodríguez B, Suárez LE, Markello RD, Shafiei G, Paquola C, Hagmann P, et al. Gradients of structure-function tethering across neocortex. Proc Natl Acad Sci U S A. 2019 Oct 15;116(42):21219-27.

68. Bercury KK, Macklin WB. Dynamics and Mechanisms of CNS Myelination. Dev Cell. 2015 Feb 23;32(4):447-58.

69. Arain M, Haque M, Johal L, Mathur P, Nel W, Rais A, et al. Maturation of the adolescent brain. Neuropsychiatr Dis Treat. 2013;9:449-61.

70. Yurgelun-Todd D. Emotional and cognitive changes during adolescence. Curr Opin Neurobiol. 2007 Apr;17(2):251-7.

71. Paquola C, Bethlehem RA, Seidlitz J, Wagstyl K, Romero-Garcia R, Whitaker KJ, et al. Shifts in myeloarchitecture characterise adolescent development of cortical gradients. Gold JI, Satterthwaite T, Mills K, Fulcher B, editors. eLife. 2019 Nov 14;8:e50482.

72. Fawcett JW, Oohashi T, Pizzorusso T. The roles of perineuronal nets and the perinodal extracellular matrix in neuronal function. Nat Rev Neurosci. 2019 Aug;20(8):451-65.

73. Liu F, Duan Y, Peterson BS, Asllani I, Zelaya F, Lythgoe D, et al. Resting state cerebral blood flow with arterial spin labeling MRI in developing human brains. European Journal of Paediatric Neurology. 2018 Jul 1;22(4):642-51.

74. Dosenbach NUF, Fair DA, Miezin FM, Cohen AL, Wenger KK, Dosenbach RAT, et al. Distinct brain networks for adaptive and stable task control in humans. PNAS. 2007 Jun 26;104(26):11073-8.

75. Janfaza M, Sherman TI, Larmore KA, Brown-Dawson J, Klein KO. Estradiol levels and secretory dynamics in normal girls and boys as determined by an ultrasensitive bioassay: a 10 year experience. J Pediatr Endocrinol Metab. 2006 Jul;19(7):901-9.

76. Krause DN, Duckles SP, Gonzales RJ. Local estrogenic/androgenic balance in the cerebral vasculature. Acta Physiol (Oxf). 2011 Sep;203(1):181-6.

77. Gündling P, Haneder J, Gaab MR. Correlation Between CBF and pCO2, pO2, pH, Hemoglobin, Blood Pressure, Age, and Sex. In: Hartmann A, Hoyer S, editors. Cerebral 
Blood Flow and Metabolism Measurement [Internet]. Berlin, Heidelberg: Springer; 1985 [cited 2021 Jun 25]. p. 51-5. Available from: https://doi.org/10.1007/978-3-642-70054-5_6

78. Liu Y, Zhu X, Feinberg D, Guenther M, Gregori J, Weiner MW, et al. Arterial spin labeling MRI study of age and gender effects on brain perfusion hemodynamics. Magn Reson Med. 2012 Sep;68(3):912-22.

79. Berman KF, Schmidt PJ, Rubinow DR, Danaceau MA, Van Horn JD, Esposito G, et al. Modulation of cognition-specific cortical activity by gonadal steroids: A positron-emission tomography study in women. Proc Natl Acad Sci U S A. 1997 Aug 5;94(16):8836-41.

80. Chung YS, Poppe A, Novotny S, Epperson CN, Kober H, Granger DA, et al. A preliminary study of association between adolescent estradiol level and dorsolateral prefrontal cortex activity during emotion regulation. Psychoneuroendocrinology. 2019 Nov;109:104398.

81. Seney ML, Sibille E. Sex differences in mood disorders: perspectives from humans and rodent models. Biol Sex Differ [Internet]. 2014 Dec 7 [cited 2021 May 15];5. Available from: https://www.ncbi.nlm.nih.gov/pmc/articles/PMC4268901/

82. Dhamala E, Jamison KW, Sabuncu MR, Kuceyeski A. Sex classification using long-range temporal dependence of resting-state functional MRI time series. bioRxiv. 2019 Oct $21 ; 809954$.

83. Sylvester CM, Corbetta M, Raichle ME, Rodebaugh T, Schlaggar BL, Sheline YI, et al. Functional network dysfunction in anxiety and anxiety disorders. Trends Neurosci. 2012 Sep;35(9):527-35.

84. Kaiser RH, Andrews-Hanna JR, Wager TD, Pizzagalli DA. Large-scale network dysfunction in Major Depressive Disorder: Meta-analysis of resting-state functional connectivity. JAMA Psychiatry. 2015 Jun 1;72(6):603-11.

85. Baller EB, Kaczkurkin AN, Sotiras A, Adebimpe A, Bassett DS, Calkins ME, et al. Neurocognitive and functional heterogeneity in depressed youth. Neuropsychopharmacology. 2020 Oct 2;

86. Fitzgerald KD, Liu Y, Stern ER, Welsh RC, Hanna GL, Monk CS, et al. Reduced ErrorRelated Activation of Dorsolateral Prefrontal Cortex Across Pediatric Anxiety Disorders. J Am Acad Child Adolesc Psychiatry. 2013 Nov;52(11):1183-1191.e1.

87. Satterthwaite TD, Wolf DH, Erus G, Ruparel K, Elliott MA, Gennatas ED, et al. Functional maturation of the executive system during adolescence. Journal of Neuroscience. 2013;33(41):16249-61.

88. Gilbert DL, Huddleston DA, Wu SW, Pedapati EV, Horn PS, Hirabayashi K, et al. Motor cortex inhibition and modulation in children with ADHD. Neurology. 2019 Aug 6;93(6):e599-610. 
89. Schulte T, Hong J-Y, Sullivan EV, Pfefferbaum A, Baker FC, Chu W, et al. Effects of age, sex, and puberty on neural efficiency of cognitive and motor control in adolescents. Brain Imaging Behav. 2020 Aug;14(4):1089-107.

90. Wang J, Licht DJ, Jahng G-H, Liu C-S, Rubin JT, Haselgrove J, et al. Pediatric perfusion imaging using pulsed arterial spin labeling. J Magn Reson Imaging. 2003 Oct;18(4):40413.

91. Proisy M, Bruneau B, Rozel C, Tréguier C, Chouklati K, Riffaud L, et al. Arterial spin labeling in clinical pediatric imaging. Diagn Interv Imaging. 2016 Feb;97(2):151-8.

92. Ye FQ, Berman KF, Ellmore T, Esposito G, van Horn JD, Yang Y, et al. H(2)(15)O PET validation of steady-state arterial spin tagging cerebral blood flow measurements in humans. Magn Reson Med. 2000 Sep;44(3):450-6.

93. Tong Y, Hocke LM, Lindsey KP, Erdoğan SB, Vitaliano G, Caine CE, et al. Systemic LowFrequency Oscillations in BOLD Signal Vary with Tissue Type. Front Neurosci. 2016 Jun $30 ; 10: 313$.

94. Yu-Feng Z, Yong H, Chao-Zhe Z, Qing-Jiu C, Man-Qiu S, Meng L, et al. Altered baseline brain activity in children with ADHD revealed by resting-state functional MRI. Brain and Development. 2007;29(2):83-91.

95. Bu X, Hu X, Zhang L, Li B, Zhou M, Lu L, et al. Investigating the predictive value of different resting-state functional MRI parameters in obsessive-compulsive disorder. Transl Psychiatry. 2019 Jan 17;9(1):1-10.

96. Shanmugan S, Wolf DH, Calkins ME, Moore TM, Ruparel K, Hopson RD, et al. Common and dissociable mechanisms of executive system dysfunction across psychiatric disorders in youth. Am J Psychiatry. 2016 01;173(5):517-26.

97. Wade M, Zeanah CH, Fox NA, Nelson CA. Global deficits in executive functioning are transdiagnostic mediators between severe childhood neglect and psychopathology in adolescence. Psychol Med. 2020 Jul;50(10):1687-94. 